\title{
Combined radiochemotherapy for postoperative recurrence of oesophageal cancer
}

\author{
J-L Raoul, E Le Prisé, B Meunier, V Julienne, P-L Etienne, M Gosselin, B Launois
}

\begin{abstract}
Postoperative recurrences are common after resection for oesophageal cancer. From January 1986 to September 199331 patients (30 males, one female, mean (SD) age: $57.5(8.8)$ years) were treated for locoregional recurrence $(n=24)$, metastases $(n=6)$ or both $(n=1)$ occurring $15 \cdot 0$ (12.6) months after initial surgery. Radiotherapy and chemotherapy were combined in all cases. Symptomatic improvement was seen in 23 cases $(74 \%)$ and lasted (excluding treatment period) for 6.3 (4) months. Objective tumoral response was seen in 20 patients $(65 \%)$ including eight $(26 \%)$ complete responses. Survival rates were at respectively six months, one, two, and three years: $\mathbf{7 0 . 7 \%}$, $47 \cdot 1 \%, 17 \cdot 1 \%, 4 \cdot 3 \%$. In conclusion, these results show that combined therapy could have a beneficial symptomatic effect and can be associated with prolonged survival in patients with postoperative recurrences of oesophageal cancer.

(Gut 1995; 37: 174-176)
\end{abstract}

Keywords: oesophageal cancer, radiochemotherapy.

Whenever possible, surgical resection remains the reference treatment for both squamous cell carcinoma and adenocarcinoma of the oesophagus. Even in operable cases, however, recurrent local or regional invasion often recurs after resection, reducing five year survival to less than $25 \%$. Several combinations of first line radiotherapy and chemotherapy have been proposed as adjuvants to surgery but, although complete histological response can be achieved in 25 to $42 \%$ of the cases, ${ }^{1}$ no one combination has been found to produce better outcome than surgical resection alone. ${ }^{2}$ Consequently, until recently, when cancer of the oesophagus continues to spread after surgery, most groups propose either simple symptomatic measures aimed at reducing dysphagia or radiotherapy alone. In an attempt to improve survival, we therefore gave combined radiochemotherapy in cases of recurrent postoperative cancer of the oesophagus.

Service de Chirurgie Digestive, CHRU

Pontchaillou, Rennes,

France

B Meunier

B Launois

Correspondence to: Dr J-L Raoul, Centre E Marquis, rue de la Bataille Flandres, Dunkerque BP 6279, 35062 Rennes Cédex, France.

Accepted for publication 7 December 1994

\section{Methods}

From January 1986 to September 1993, 31 patients (30 males, one female; mean (SD) age $57.5(8.8)$ years) received combined radiochemotherapy for recurrent squamous cell carcinoma of the oesophagus after initial surgical resection. Alcohol and smoking were risk factors in 27 and one had been treated for another cancer (tongue $=1$ ). The tumour was located in the middle third of the oesophagus in 18 cases, in the lower third in 10 cases, and in the upper third in three. Surgical resection had been performed in 16 of the patients with thoracotomy and in 15 without thoracotomy. Surgical specimens of the tumours were scored according to the AJCC/UICC pathology classification. ${ }^{3}$ There were three in stage $I, 15$ in stage IIA, two in stage IIB, and 11 in stage III. The pathologist reported evidence of invasion of the resection margin in nine cases.

In most patients, dysphagia was the first sign of recurrence $(n=7)$. Others complained of dysphonia $(n=6)$, chest pain $(n=4)$ or cough $(n=3)$. Physical examination revealed palpable cervical lymph nodes as the first sign in six patients. General health status, as defined by the WHO, was good or excellent (stage 0, $n=17$; stage $1, n=14$ ) at diagnosis of recurrent oesophageal cancer. In most patients, body weight had remained unchanged $( \pm 15 \%$ of weight at initial diagnosis). Weight loss had exceeded $15 \%$ in only three. Recurrent local or regional invasion involving lymph nodes, the anastomoses or the gastric plasty was seen in 24 cases including two with several localisations (Table I). Metastasis was found in six and both local or regional invasion and metastasis occurred in one. The diagnosis of recurrent postoperative oesophageal cancer was based on pathological examination of biopsy specimens in 24 cases. Imaging and the clinical course were sufficient for diagnosis in the other seven cases. The mean (SEM) delay between initial surgical resection and recurrence was $15.0(12 \cdot 6)$ months (range 2 to 49 months).

Radiotherapy and chemotherapy were combined in all cases. The chemotherapy protocol included cisplatin $\left(100 \mathrm{mg} / \mathrm{m}^{2}\right.$ on day 1$)$ and continuous infusion of 5-fluorouracil (600 $\mathrm{mg} / \mathrm{m}^{2} /$ day from day 2 to day 5 ). Usually, a total radiotherapy dose of $60 \mathrm{~Gy}$ was given from anterior and posterior fields in 30 fractions with a $25 \mathrm{MeV}$ linear accelerator. In eight patients, 45 Gy was given in 25 fractions. The

TABLE I Localisations of recurrent oesophageal cancer after surgical resection

\begin{tabular}{lc}
\hline Localisation & Number \\
\hline Local regional invasion & 24 \\
Lymph node & 15 \\
Anastomosis & 5 \\
Gastric plasty & 2 \\
Multiple & 2 \\
Metastasis & 6 \\
Lung & 3 \\
Liver & 2 \\
Bone & 1 \\
Local regional invasion and metastasis & 1 \\
\hline
\end{tabular}


TABLE II Complications of radiochemotherapy after surgical resection for cancer of the oesophagus

\begin{tabular}{ll}
\hline Complication & Number \\
\hline Considerable weight loss & 3 \\
Haematological disorders & 2 \\
Severe mucositis & 2 \\
Sepsis & 1 \\
Renal failure & 1 \\
Suicide & 1 \\
\hline
\end{tabular}

radiation fields comprised the mediastinum and macroscopically invaded areas. Generally (21 cases), the combination protocol was given sequentially: chemotherapy (5-fluorouracil+cisplatin) followed by radiotherapy ( 30 Gy in 15 fractions) then a second chemotherapy session followed again with $30 \mathrm{~Gy}$. In case of incomplete response or a second recurrence after complete remission, and if the patient's general health permitted, chemotherapy sessions were again performed. One patient thus underwent nine sessions combining platinium and 5-fluorouracil.

Endoscopic therapy was required in 17 patients, with several dilatations in 15 . Respiratory digestive fistulas required insertion of respiratory tract prostheses in five patients, including two who also required digestive tract prostheses.

\section{Results}

Tolerance was considered good in 21 patients. Complications were seen in 10 (Table II).

Clear symptomatic response was achieved in 23 patients $(74 \%)$ including six in whom the symptoms disappeared totally. In five patients, there was no change in symptomatology and in three clinical signs worsened during treatment. Excluding the treatment period, symptomatic improvement lasted for 6.3 (4) months (range 2 to 18 months). Weight gain occurred in 16 patients. Objective tumoral response was seen in 20 patients $(65 \%)$. Among these, eight $(26 \%)$ reached complete remission as objectively assessed clinically or by imaging.

At the end of the study, 26 patients had died. Mean follow up for the five surviving patients was 14 months.

The survival rate (Kaplan-Meier method), taking as the starting point the diagnosis of recurrence, was $70 \cdot 7 \%$ at six months, $47 \cdot 1 \%$ at one year, $17 \cdot 1 \%$ at two years, and $4.3 \%$ at

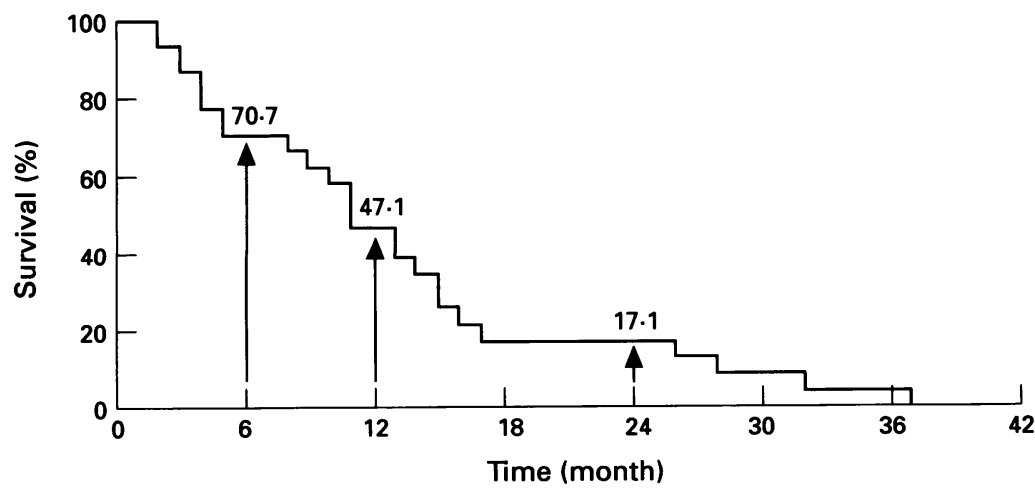

Survival rates (Kaplan-Meier method) of our series of 31 postoperative recurrences of oesophageal cancer treated by combined chemoradiotherapy. Median survival time $=10.7$ months. three years (Figure). Median survival time was 10.7 months. Taking the initial diagnosis of cancer of the oesophagus as the starting point, survival rate was $86.8 \%$ at one year, $55.6 \%$ at two years, $31 \cdot 8 \%$ at three years, $19 \cdot 1 \%$ at four years, and $4.8 \%$ at five years. Median survival time was 28.6 months.

In our study, factors that showed no effect on survival rate were: initial pathological classification of the tumour (stage I+IIA + IIB, median 11.7 months; stage III, median 9.6 months), delay between initial surgical resection and recurrence ( $\geqslant 12$ months, median $=12.6$ months; $<12$ months, median 9.4 months), patient age ( $\geqslant 57$ years, median 9.8 months; $<57$ years, median 11.4 months), and the local or metastasic nature of the recurrence (local or regional invasion, median 10 months; metastasis, median 11.8 months). Inversely, objective tumour response to treatment was found to be a factor predictive of outcome $(p<0.02)$. Median survival time in responders was 12.9 months compared with 3.5 months in non-responders. Survival rates at one, two, and three years were $63.9,21.3$, and $7 \cdot 1 \%$ respectively in responders and $18 \cdot 2$, $9 \cdot 1$, and $0 \%$ respectively in non-responders.

\section{Discussion}

This study shows that medical treatment, mainly combined chemotherapy and radiotherapy, can provide a certain improvement in terms of survival and symptomatology in a selected population of patients with recurrent cancer of the oesophagus after initial surgical resection.

The serious prognosis of oesophageal cancer is directly related to the frequency of postoperative recurrence. Longterm results in all the series in published works have been disappointing as reported median survival rarely exceeds 18-20 months and survival at five years is about $10 \%{ }^{4}$ Prognosis is better if the surgeon can achieve total curative resection, but even in these cases, five year survival is not much longer, $23(7) \% .{ }^{4}$ This poor prognosis is the consequence of rapid recurrence either because of local and regional invasion or distant metastasis.

Few authors have reported the localisation of recurrent lesions. Peracchia et al ${ }^{5}$ reported a series of 240 patients with a median survival of 20.6 months and a five year survival of $20.8 \%$. In this series, local invasion was the most frequent $(43 \%)$, followed by metastasis $(33 \%)$, both local invasion and metastasis were seen in $24 \%$ of the cases. In another series of patients treated with radiotherapy, ${ }^{6}$ computed tomography was shown to be effective in identifying local or distant recurrence after oesophagectomy in 58 patients (squamous cell carcinoma $=21$, adenocarcinoma $=36$, anaplasic tumour $=1$ ) who had received combined chemo and radiotherapy preoperatively. In this series, local or regional invasion predominated (extragastric mass or invasion of the mediastinal lymph nodes 16; metastasis to lung, liver, pleura, adrenal glands 23; but also invasion of the gastric plasty 11 , particularly in 
the adenocarcinoma cases). The high rate of local-regional invasion can be explained by the presence of neoplasic tissue remaining after surgery.

In a large necropsy series of oesophageal cancers, ${ }^{7} 11$ patients died early in the postoperative period. Among these 11 cases, three still had residual tumoral tissue after oesophageal resection. This finding is similar to the fact that, in our series, the pathology examination showed neoplasic invasion of the resection slice. In addition, in several cases, recurrence came very early after the operation, suggesting that neoplasic tissue, left in situ, continued to develop after surgery, invading surrounding tissues. In a report of extensive lymph node dissection, the percentage of distant lymph node involvement was extremely high even in cases with tumours considered to be superficial ${ }^{8}$ as invaded lymph nodes were found in $50 \%$ of the patients classes pT1.

It seems therefore that combining radiotherapy and chemotherapy could improve the therapeutic effect on local and local-regional invasion. But, in a series of 42 patients $^{9}$ who were given a combined radiochemotherapy followed by surgery protocol, recurrence developed in 16, including one oesophagus, four oesophagus and distant metastasis, and 11 with metastasis alone.

Few treatment protocols for these postoperative recurrences of oesophageal cancer have been described in published reports. Usually, radiotherapy or endoscopic techniques (repeated dilatations, prosthesis, local alcohol injection) have been used. The promising results reported for radiochemotherapy combinations for cancers of the oesophagus ${ }^{10-13}$ encouraged us to routinely propose, in cases of recurrence, such combined treatment for all patients whose general health permitted further treatment. The more recent finding by Herskovic et al ${ }^{14}$ of the greater effect of combined radiochemotherapy over radiotherapy alone, confirms the importance of this new approach.

Despite the modest results obtained, our series would suggest certain promising effects. The median survival in our population was 10.7 months, not very different from survival rates obtained with first line radiochemotherapy combinations given as the only treatment. ${ }^{9-12} 14$ But in these studies, the initial tumoral stage was less advanced than in our series because patients with metastasis had been excluded. Our observed survival rate is better than those obtained after surgical resection performed as macroscopically palliative treatment ${ }^{4}{ }^{15}$ or after potentially curative resection in patients with invaded lymph nodes. ${ }^{16}$ The population we report here, of course, does not represent all the patients we have seen for postoperative recurrence, but our inclusion criteria (age, general health, renal function, haematological or liver status) were not different from the criteria usually used in reported prospective medical or surgical series. In any case, even if we cannot state that we have improved survival in these patients, our findings would suggest that this protocol did have a beneficial effect for most of our patients. First of all, symptomatology was improved in $75 \%$ of the patients for a mean duration of more than six months (treatment period excluded). Secondly, we saw a $65 \%$ tumour response rate including $26 \%$ with complete response based on clinical or imaging criteria, or both. Finally, survival in patients with an objective response to treatment was clearly better than in nonresponders (12.9 $v 3.5$ months).

In conclusion, our findings show that combining chemotherapy and radiotherapy does have a beneficial symptomatic effect in patients with recurrence of squamous cell carcinoma of the oesophagus. Tolerance to such treatment is good. Its effectiveness has been shown by a noticeable improvement in symptomatology and by median survival comparable with that obtained after palliative resection. These results also emphasise the need for close follow up in patients who have had surgical resection for cancer of the oesophagus.

1 Dougherty JB, Coia LR, Skinner DB. Multimodality therapy of esophageal and gastric cancer. ASCO Educational book, 1993: 173-83.

2 Le Prisé E, Etienne PL, Meunier B, Maddern G, Ben Hassel M, Gédouin D, et al. A randomized study of chemotherapy, radiation therapy, and surgery versus chemotherapy, radiation therapy, and surgery versus surgery for localized squamous cell carc

3 International Union Against Cancer. Hermanek P, Sobin LH, eds. TNM classification of malignant tumours. 4 th ed. Berlin: Springler-Verlag, 1987

4 Muller JM, Erasmi H, Stelzner M, Zieren U, Pichlmaier H. Surgical therapy of oesophageal carcinoma. Br $\mathcal{f}$ Surg 1990; 77: 845-57.

5 Peracchia A, Bardini R, Castoro C, Sorrentino P, Segalin A, Ruol A, et al. La lymphadénectomie dans le traitement du cancer de l'oesophage intrathoracique. Ann Chir 1990; 44: 9-17.

6 Carliste JG, Quint LE, Francis IR, Orringer MB, Smick JF, Gross BH. Recurrent esophageal carcinoma: CT evaluation after esophagectomy. Radiology 1993; 189: 271-5.

7 Mandard AM, Chasle J, Marnay J, Villedieu B, Bianco C, Roussel A, et al. Autopsy findings in 111 cases of esophageal cancer. Cancer 1981; 48: 329-35.

8 Kato H, Tachimori Y, Mizobuchi S, Igaki H, Ochiai A. Cervical, mediastinal, and abdominal lymph node dissection (three-field dissection) for superficial carcinoma of the thoracic esophagus. Cancer 1993; 72: 2879-82.

9 Gill PG, Denham JW, Jamieson GG, Devitt PG, Yeoh E, Olweny C. Patterns of treatment failure and prognostic factors associated with the treatment of esophageal carcinoma with chemotherapy and radiotherapy either as sole treatment or followed by surgery. F Clin Oncol 1992; 10: $1037-43$.

10 Leichman L, Herskovic A, Leichman CG, Lattin PB Steiger Z, Tapazoglou E, et al. Nonoperative therapy for squamous-cell cancer of the esophagus. $\mathcal{F}$ Clin Oncol 1987; squamous-cell $365-70$.

11 Chan A, Wong A, Arthur K. Concomitant 5-fluorouracil infusion, mitomycin $C$ and radical radiation therapy in esophageal squamous cell carcinoma. Int $\mathcal{f}$ Radiat Oncol Biol Phys 1989; 16: 59-65.

12 Coia LR, Engstrom PF, Paul AR, Stafford PM, Hanks GE Long-term results of infusional 5-FU, mitomycin $\mathrm{C}$, and radiation as primary management of esophageal carcinoma. Int $\mathcal{F}$ Radiat Oncol Biol Phys 1991; 20: 29-36.

13 Forastiere A, Orringer MB, Perez-Tamayo C, Urba SG, Zahurak M. Preoperative chemoradiation followed by transhiatal esophagectomy for carcinoma of the esophagus: final report. $\mathcal{f}$ Clin Oncol 1993; 11: 1118-23.

14 Herskovic A, Martz K, Al-Sarraf M, Leichman L, Brindle J, Vait Kevicius $\mathrm{V}$, et al. Combined chemotherapy and radiotherapy compared with radiotherapy alone in patients with cancer of the esophagus. N Engl f Med 1992; 326: 1593-8

15 Launois B, Paul JL, Lygidakis NJ, Campion JP, Malledant Y, Grossetti D, et al. Results of the surgical treatment of carcinoma of the esophagus. Surg Gynecol Obstet 1983; 156: 753-60.

16 Goldminc M, Maddern G, Le Prise E, Meunier B, Campion JP, Launois B. Oesophagectomy by a transhiatal approach or thoracotomy: a prospective randomized trial. Br F Surg 1993; 80: 367-70. 